\title{
CORRECTION
}

\section{Antagonistic acetylases}

Guillaume Tena

Nature Plants 1, 15084 (2015); published online 2 June 2015; corrected 4 June 2015

In the version of this Research Highlight originally published, there was a typographical error in the final sentence. It should have read 'The study by Xin Li and co-workers highlights the double role of $\mathrm{N}$-terminal acetylation in protein stability in general, but also specifically in immunity.' 